S.K. PATRA

G. MOHAN RAO

\title{
Synthesis of carbon nanotubes by ECR plasma-assisted chemical vapor deposition
}

\author{
Department of Instrumentation, Indian Institute of Science, Bangalore 560012, India
}

\begin{abstract}
Carbon nanotubes have been grown using an electron cyclotron resonance (ECR) plasma source at a substrate temperature of $500{ }^{\circ} \mathrm{C}$. Methane has been used as the source gas. A network of carbon nanotubes has been observed in scanning electron microscopy. Transmission electron microscopy revealed that the structure consists of straight, Y-junction and ring-like nanotubes. Further, electron diffraction of the nanotubes confirms a graphite crystal structure.
\end{abstract}

PACS 81.16.He; 68.37.Lp; 68.37.Hk; 85.35.Kt; 75.75.+a

\section{$1 \quad$ Introduction}

Since the first observation of carbon nanotubes (CNTs) [1], they have attracted great attention due to their potential application in electron devices [2], flat-panel displays [3], hydrogen storage [4] and scanning probes [5]. Highquality single- or multi-walled carbon nanotubes have been deposited using several techniques like high-pressure arcs [6], laser ablation [7], chemical vapor deposition (CVD) [8] and plasma-enhanced CVD [9]. Though microwave plasma and $\mathrm{rf}$ plasma have been extensively used for the synthesis of carbon nanotubes, electron cyclotron resonance (ECR) plasma CVD has not been so widely used for this purpose, though high plasma density in the ECR source could result in interesting studies.

So far, basically three kinds of nanotube structures have been reported, viz. straight, Y junction and ring-like. For different kinds of applications, different types of structures are required. Straight and vertically aligned nanotubes find application in field-emission electron sources [10]. For nanodevices, it is necessary to connect different nanotubes of different diameters and chirality to get metal-semiconductor, metal-metal or semiconductor-semiconductor junctions $[11,12]$ and, for nano-electronics, a complex three-point nanotube junction ( $\mathrm{T}$ and $\mathrm{Y}$ ) has been proposed as the building block. The Y- and T-type junctions appear to defy the conventional models in favor of an equal number of fiveand seven-membered rings. However, connection of carbon nanotubes together to form a junction is difficult. The first observation of branching carbon nanotubes to demonstrate the formation of L, T and Y junctions was reported in an arc discharge method [13]. Pyrolysis of an organometallic precursor along with thiamine [14] at $1100{ }^{\circ} \mathrm{C}$ and by pyrolysis of methane at $1000{ }^{\circ} \mathrm{C}$ over cobalt supported on magnesium oxide [15] have been reported to synthesize Y junctions. Another kind of structure that has been observed is in the form of a ring. It has been predicted theoretically that this kind of structure possesses a giant diamagnetic moment as its strength is three orders of magnitude stronger than that of the diamagnetic moment of graphite at a 0.1-T field [16].

In the present study, we synthesized all the abovementioned structures using ECR plasma at a substrate temperature as low as $500{ }^{\circ} \mathrm{C}$, and the results are presented in this communication.

\section{$2 \quad$ Experimental}

A home-built ECR plasma source [17] has been used to grow carbon nanotubes. Carbon nanotubes were prepared on nickel-coated Si substrates by decomposing methane. Initially, a nickel film about 40-nm thick was deposited on a $\mathrm{Si}(100)$ substrate at room temperature by magnetron sputtering at a working pressure of $10^{-3}$ mbar (argon) while the base pressure of the system was $10^{-5}$ mbar. The carbon nanotubes were grown on the nickel film by decomposing methane $\left(\mathrm{CH}_{4}\right)$ using ECR plasma $(500 \mathrm{~W})$ at a substrate temperature of $500^{\circ} \mathrm{C}$. The working gas pressure was maintained at $6 \times 10^{-4}$ mbar by using a mass-flow controller with a flow rate $10 \mathrm{sccm}$. A flat resistive heater has been used to heat the substrate and the temperature of the substrate was maintained within $5{ }^{\circ} \mathrm{C}$ of the set temperature. The growth time for carbon nanotubes was $30 \mathrm{~min}$. A black coating was observed on silicon. The morphology of the carbon nanotubes was studied by scanning electron microscopy (JSM-840 scanning electron microscope), and structural analysis was carried out using transmission electron microscopy (JEOL 2000 FX II ••). Carbon nanotubes were dispersed in methanol with the help of an ultrasonator. A few drops of suspension were then deposited on an amorphous holey carbon grid to carry out TEM investigation of the sample.

\section{3}

\section{Results and discussion}

Figure 1 shows electron micrographs of nickelcoated silicon substrates. It shows round-shaped nickel 


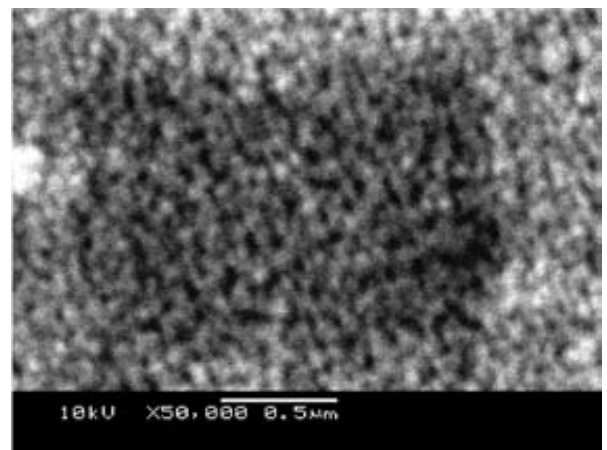

FIGURE 1 Scanning electron micrograph of nickel nanoparticles

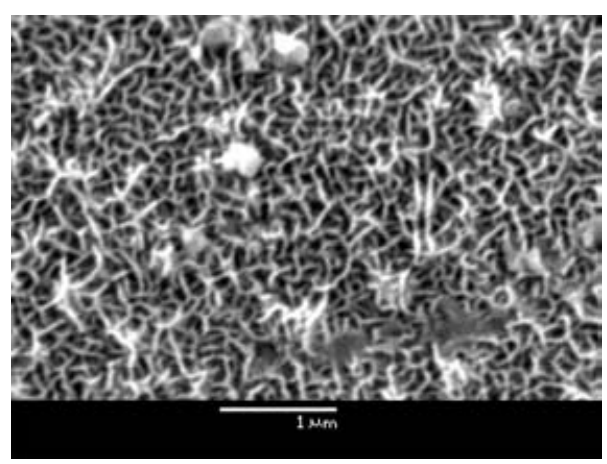

FIGURE 2 Low-magnification SEM image of CNTs

nanoparticles of about 50-nm diameter that are evenly distributed. The carbon nanotubes nucleate and grow on these catalytic Ni particles and their diameter depends on the size of the Ni nanoparticles [18]. The electron micrograph (Fig. 2) shows a three-dimensional network of carbon nanotubes. High-magnification SEM images, as shown in Fig. 3, show that the material consists of a network of nanotubes and the diameter of the tubes is in the range of $40-65 \mathrm{~nm}$. These nanotubes are curly and short in length. This can be attributed to the low substrate temperature $\left(500^{\circ} \mathrm{C}\right)$ during growth. Figure 4 shows a transmission electron micrograph of carbon nanotubes. Hollowness can be clearly seen from the image, with Ni nanoparticles attached to one end of the nanotubes. Carbon contamination on nickel can also be observed. This can be attributed to the fact that only methane gas has been used as the source gas without diluting it in hydrogen or ammonia.

Figure 5 shows the presence of Y-junction nanotubes. The angle between the two arms is equal to $90^{\circ}$. The bright features observed at the junction could be due to carbon contamination. A ring-shaped nanostructure is also observed. Because of their geometry, they appear ideal candidates for enhancing the effect of the interplay between the de-localized $\pi$ electrons and the geometrical structure of the torus, and thus enhance the magnetic response in the presence of a magnetic field. So far there are few reports on the observation of ring-shaped carbon nanotubes [19-21]. Liu et al. [19] reported the observation of nanotube rings and these rings had a typical diameter of 300-500 $\mathrm{nm}$ and the thickness in the range of 5-15 $\mathrm{nm}$. On the theoretical side carbon toroids can be considered either by introducing curvature-inducing defects as in the helical nanotubes or by bending elastically a straight nanotube so that it closes itself [22]. Figure 6 shows a TEM micrograph of ring-shaped carbon nanotubes. The image shows that the

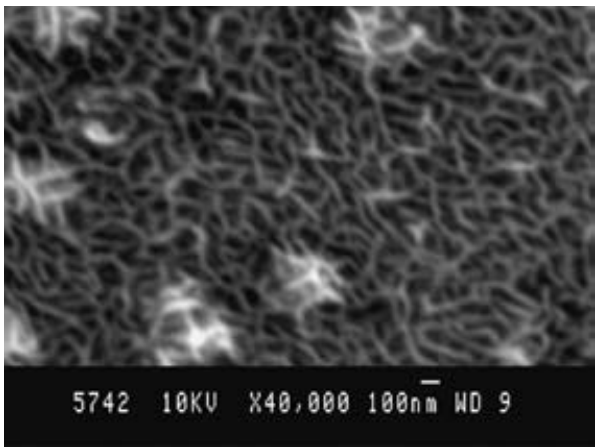

FIGURE 3 High-magnification SEM image

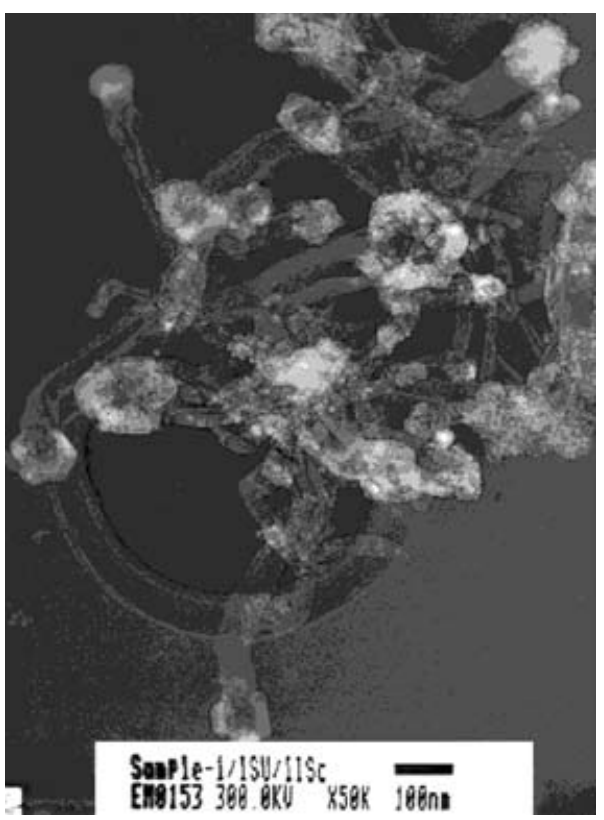

FIGURE 4 TEM image of CNTs

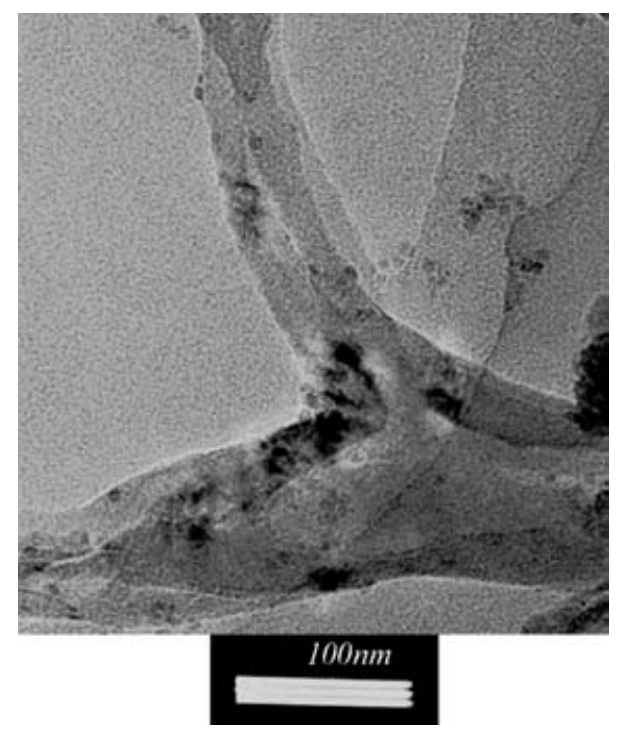

FIGURE 5 Y-junction CNT

rings do not have a constant thickness around the circumference. It looks as though they are formed by separate ropes being curled together. Low magnification of the TEM image and strong background noise from the holey carbon film make 


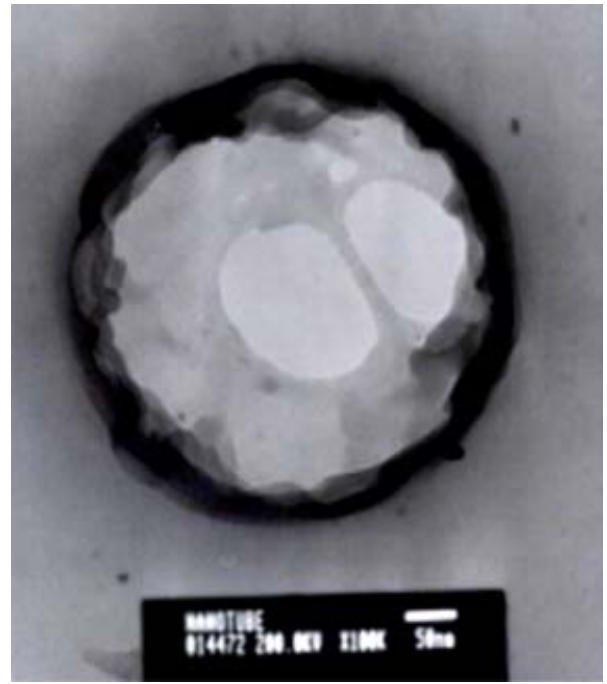

FIGURE 6 TEM image of ring-shaped structure

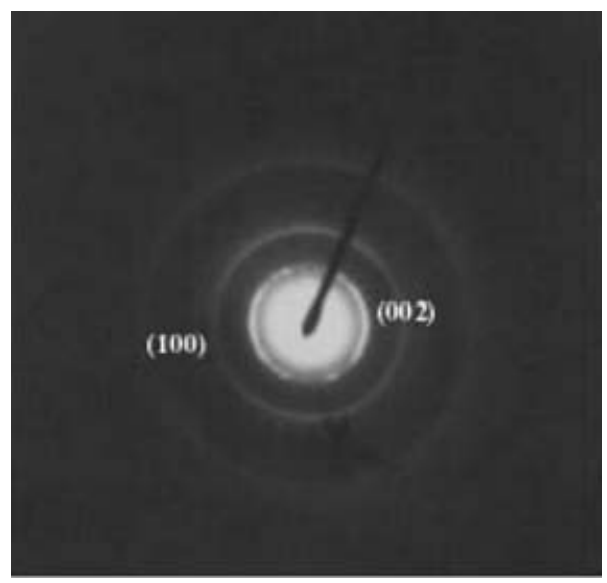

FIGURE 7 Electron-diffraction pattern

it difficult to distinguish the tubular nature of the ring. The diameter of the ring is around $450 \mathrm{~nm}$ while the thickness is $15-20 \mathrm{~nm}$. The electron-diffraction pattern of the carbon nanotubes is shown in Fig. 7. It shows diffraction rings corresponding to graphite (002) and (100) planes, confirming the graphite crystal structure of the nanotubes. The Bragg spacings $d_{002}$ and $d_{100}$ were found to be $0.347 \mathrm{~nm}$ and $0.210 \mathrm{~nm}$ as compared to $0.348 \mathrm{~nm}$ and $0.212 \mathrm{~nm}$ respectively for singlecrystal graphite (JCPDS data).

All the three morphologies of carbon nanotubes were observed in the same sample. Though the majority of the carbon nanotubes were straight, Y-junction and circular nanotubes were observed regularly during TEM investigation. It is not possible to say whether they are evenly distributed and what their percentage is, as it was not possible to separate them from the lump, as the carbon nanotubes are very short in length and also the resolution of the microscope was not very good.

\section{Conclusions}

In the present study, we have shown that ECR plasma can be used to grow carbon nanotubes at a nominal substrate temperature of $500^{\circ} \mathrm{C}$. We have observed all three kinds of morphologies of carbon nanotubes. Though quantitative analysis was not possible, it would be interesting to study the growth process of carbon nanotubes using a high-density plasma source such as an ECR plasma. It would be interesting to study the effects of substrate temperature and methane partial pressure for the growth of only one particular type of nanotube structure for effective use in an application. Our efforts are directed towards this goal.

\section{REFERENCES}

1 S. Iijima: Nature (Lond.) 354, 56 (1991)

2 R. Martel, T. Schmidt, H.R. Shea, T. Hertel, P. Avouris: Appl. Phys. Lett. 73, 2447 (1998)

3 H. Dai, N. Franklin, J. Han: Appl. Phys. Lett. 73, 1508 (1998)

4 W.A. de Heer, A. Chateline, D. Ugrate: Science 270, 1179 (1995)

5 H. Dai, J.H. Hafner, A.G. Rinzler, D.T. Colbert, R.E. Smalley: Nature 384, 147 (1996)

6 C. Journet, W.K. Master, P. Bernier, A. Loiseau, M. Lamy de la Chapelle, S. Leftrant, P. Deniard, R. Lee, J.E. Fischer: Nature (Lond.) 388, 756 (1997)

7 A. Thess et al.: Science 273, 483 (1996)

8 Z.F. Ren, Z.P. Huang, J.W. Xu, J.H. Wang, P. Bush, M.P. Siegal, P.N. Provencio: Science 282, 1105 (1998)

9 C. Brower, W. Zhu, S. Jin, O. Zhou: Appl. Phys. Lett. 77, 830 (2000)

10 K.B. Teo, M. Chhowalla, G.A. Amartunga, W.I. Milne: Appl. Phys. Lett. 80, 2011 (2002)

11 L. Kouwenhoven: Science 275, 1896 (1997)

12 J.-C. Charlier, T.W. Ebessen, P. Lambin: Phys. Rev. B 53, 11108 (1996)

13 D. Zhou, S. Seraphin: Chem. Phys. Lett. 238, 286 (1995)

14 B.C. Satishkumar, P. John Thomas, A. Govindaraj, C.N.R. Rao: Appl. Phys. Lett. 77, 2530 (2000)

15 W.Z. Li, J.G. Wen, Z.F. Ren: Appl. Phys. Lett. 79, 1879 (2001)

16 L. Liu, G.Y. Guo, C.S. Jayanthi, S.Y. Wu: Phys. Rev. Lett. 88, 217206 (2002)

17 K. Deenamma Vargheese, G. Mohan Rao: Rev. Sci. Instrum. 71, 467 (2000)

18 C. Bower, O. Zhou, W. Zhu, D.J. Werder, S. Jin: Appl. Phys. Lett. 77, 2767 (2000)

19 J. Liu, H. Dai, J.H. Hafner, D.T. Colbert, R.E. Smalley, S.J. Tans, C. Dekker: Nature (Lond.) 385, 780 (1997)

20 M. Aslskog, E. Seynaeve, R.J.M. Vullers, C. Van Haesendock, A. Fonseca, K. Hernadi, J.B. Nagy: Chem. Phys. Lett. 300, 202 (1999)

21 M. Sano, A. Kamino, J. Okamura, S. Shinai: Science 293, 1299 (2001)

22 V. Meunier, P. Lambin, A.A. Lucas: Phys. Rev. B 57, 14886 (1998) 